\title{
Investigations on a Universal Relationship Between Optical Emission and Absorption of Complex Molecules in Liquid Solutions
}

\author{
A. Kawski, P. Bojarski, and B. Kukliński \\ Institute of Experimental Physics, University of Gdańsk, u.Wita Stwosza 57, 80-952 Gdańsk, Poland \\ Reprint requests to Prof. A. K. ul. Gen. Sikorskiego 11, 84-200 Wejherowo, Poland
}

Z. Naturforsch. 54a, 465-469 (1999); received July 19, 1999

\begin{abstract}
Based on a universal relationship between the extinction coefficient and the fluorescence intensity in their overlapping region, local temperatures $T^{*}$ higher than the ambient $T$ were determined for shortlived luminescent molecules of lifetimes from $7 \mathrm{ps}$ to $77 \mathrm{ps}$. The reason for such a local temperature $T^{*}$, which holds also during the emission process, is the non-establishment of statistical equilibrium over the vibrational levels of excited molecules. It is found that the intensity distribution in the fluorescence band depends slightly on the wavelength of the excitating light, which evidences the lack of thermal equilibrium with the vicinal surrounding.
\end{abstract}

Key words: Universal Relationship between the Absorption and Fluorescence Spectra; Local Temperature; Statistical Equilibrium.

\section{Introduction}

The universal relation beween the extinction coefficient and the fluorescence intensity is based on very general physical assumptions and is one of the most important photoluminescence regularities. As early as in the twentieths Kennard [1, 2] and then Stepanov [3, 4] dealt with that problem. The relation obtained by them can be presented in the form

$$
F(\tilde{v}) \equiv \ln \frac{f(\tilde{v})}{\tilde{v}^{3} \cdot \varepsilon(\tilde{v})}=-\frac{h c \tilde{v}}{k T}+\text { const, }
$$

where $f(\tilde{v})$ is the spectral distribution of the fluorescence intensity, $\tilde{v}$ the wavenumber, $\varepsilon(\tilde{v})$ the extinction coefficient, and $c$ is the velocity of light. The functions $F(\tilde{v})$ versus $\tilde{v}$ are straight lines, the slopes of which allow for the determination of $T$. This method gives the weighted average over the lifetime of the excited state of the luminescent molecule studied.

If the occupation density of vibrational levels in the excited state does not correspond to the temperature $T$ of the solution studied but to a higher "vibrational temperature" $T^{*}$, (1) should be written in the modified form [5]

$$
F(\tilde{v}, T) \equiv \ln \frac{f\left(\tilde{v}, T^{*}\right)}{\tilde{v}^{3} \cdot \varepsilon\left(\tilde{v}, T^{*}\right)}=-\frac{h c \tilde{v}}{k T^{*}}+\text { const. }
$$

Relation (2) has been investigated for liquid, viscous and rigid solutions [6-17]. It has been found that only in viscous and rigid solutions as well as in mixed solutions the local temperature $T^{*}$ is always higher than the ambient temperature $T$. Experimental investigations of (1) performed by us in the seventies [15-17] showed that both for polar and nonpolar luminescent molecules in polar and nonpolar liquid solvents the temperature $T^{*}$ is equal to the ambient temperature $T$ (within the $\pm 10 \mathrm{~K}$ accuracy). This concerns the case in which the decay time $\tau_{F}$ of the excited state is so long (about $10^{-9} \mathrm{~s}$ ) that thermal equilibration occurs between the luminescent and solvent molecules.

If, however, $\tau_{F}$ is comparable to the relaxation time $\tau_{\mathrm{r}}$ of the vibrational energy, emission occurring before relaxation is expected and the emission band is broadened on the long wavelength side. In this case the spectral distribution of emission intensity will be slightly dependent on the wavelength $\lambda_{\text {exc }}$ of the excitation light [18].

Recently, Sawicki, and Knox [19, 20] introduced the term of spectral temperature $T^{*}(\tilde{v})$ defined through the local slope of $F(\tilde{v})$. Equation (3) can be obtained by differentiating eq. (2) versus $\tilde{v}$ :

$$
T^{*}(\tilde{v})=-\frac{h c}{k}\left(\frac{\mathrm{d} F(\tilde{v})}{\mathrm{d} \tilde{v}}\right)^{-1} .
$$

In the present work we will show that for luminescent molecules with a very short lifetime $\tau_{F}$ comparable to that of the relaxation time $\tau_{\mathrm{r}}$ in liquid solvents of low viscosity the inequality $T^{*}>T$ is also valid. 

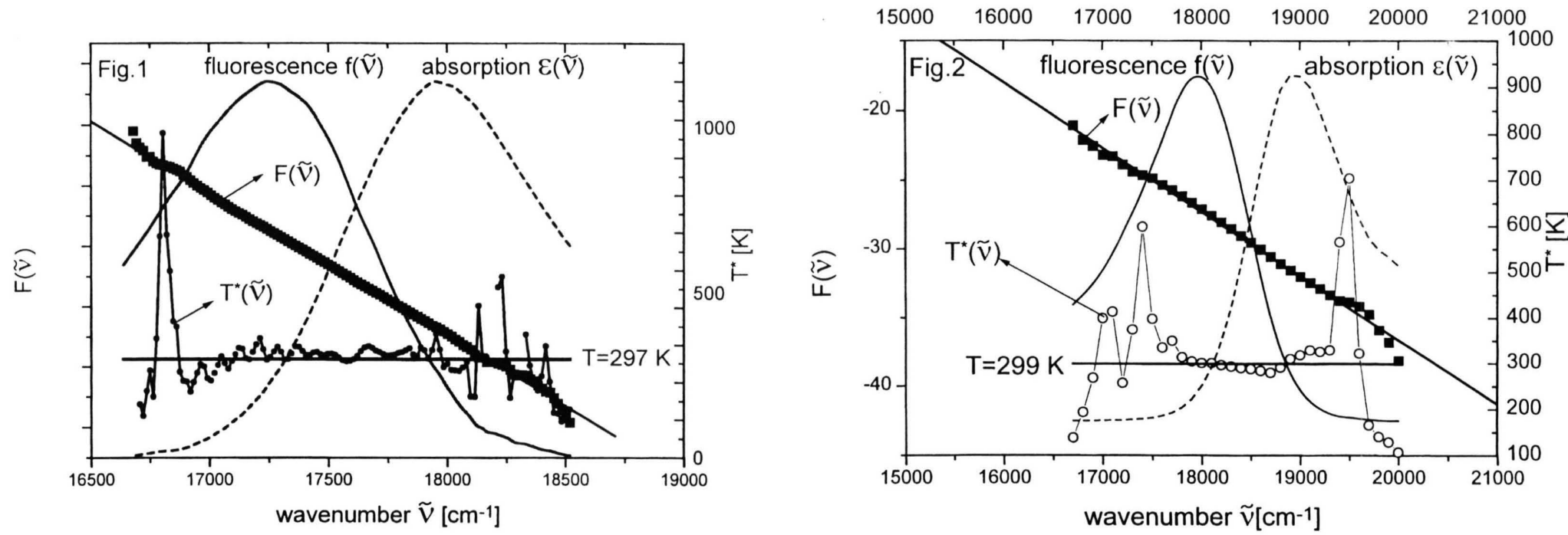

Figs. 1 and 2. Absorption, fluorescence and $T^{*}(\tilde{v})$ spectra of rhodamine $\mathrm{B}$ and rhodamine $6 \mathrm{G}$ in water $(+\mathrm{a}$ trace of $0.1 \mathrm{~N}$ solution of $\mathrm{HCl})$, respectively, as well as the corresponding $F(\tilde{v})$ function).

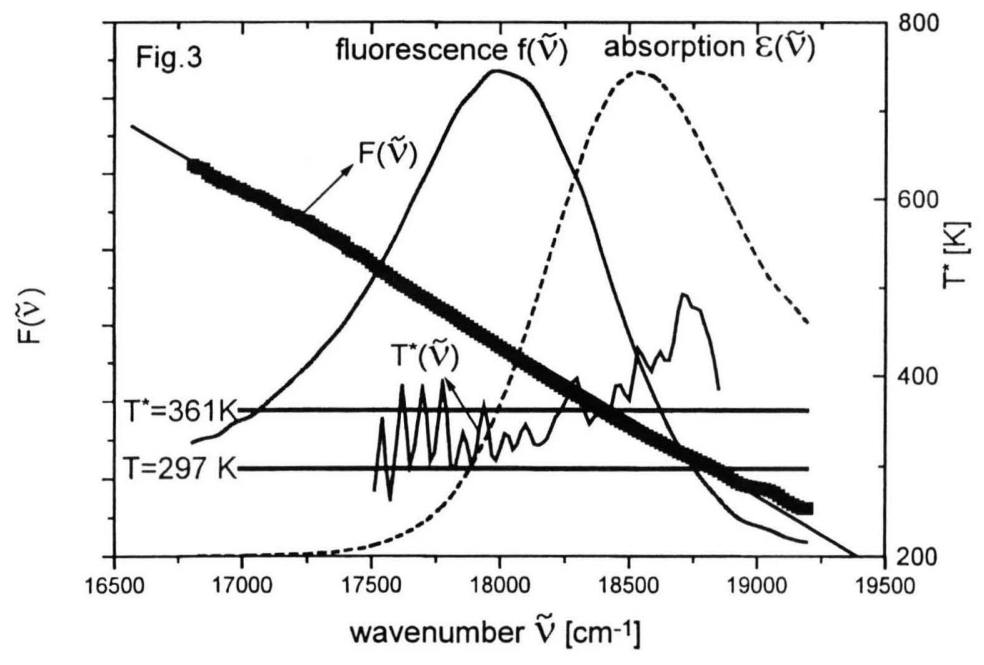

Table 1. Spectral properties and local temperatures of compounds studied in liquid solutions.

\begin{tabular}{|c|c|c|c|c|c|c|c|c|}
\hline Compound & Solvent & $\begin{array}{l}\text { Viscosity } \\
\eta \\
\left(10^{-3} \mathrm{~Pa} \mathrm{~s}\right)^{1}\end{array}$ & $\begin{array}{l}\tau_{F} \\
{\left[10^{-12}\right]}\end{array}$ & $\begin{array}{l}\bar{v}_{0-0} \\
{\left[\mathrm{~cm}^{-1}\right]}\end{array}$ & $\begin{array}{l}\tilde{v}_{\text {exc }} \\
{\left[\mathrm{cm}^{-1}\right]}\end{array}$ & $\begin{array}{l}T \\
\text { (ambient) } \\
{[\mathrm{K}]}\end{array}$ & $\begin{array}{l}T^{*} \\
{[\mathrm{~K}]}\end{array}$ & $\begin{array}{l}\Delta T^{*} \\
=T-T \\
{[\mathrm{~K}]^{2}}\end{array}$ \\
\hline Rhodamine B & water $+\mathrm{HCl}$ & & $1580[23]$ & 17614 & 18231 & 297 & 304 & 7 \\
\hline Rhodamine 6G & water $+\mathrm{HCl}$ & & $3950[23]$ & 18501 & 20000 & 299 & 309 & 10 \\
\hline Rhodamine S & water $+\mathrm{HCl}$ & 089 & ? & 17984 & 20000 & 298 & 306 & 8 \\
\hline $\mathrm{Na}$-fluorescein & water $+\mathrm{NaOH}$ & & 4270 & 20220 & 22222 & 297 & 297 & 0 \\
\hline Erythrosin B & water & & $77[24]$ & 18295 & 19608 & 297 & 361 & 64 \\
\hline \multirow[t]{2}{*}{$2 a$} & $n$-hexane & 0.326 & 7 ]25] & 27861 & 30303 & 297 & 554 & 257 \\
\hline & $n$-hexadecane & 2.33 & $20[25]$ & 28108 & 30303 & 297 & 486 & 189 \\
\hline \multirow[t]{3}{*}{$3 a$} & $n$-hexane & 0.326 & $19[25]$ & 27994 & 30303 & 297 & 402 & 105 \\
\hline & $n$-deca & 0.818 & $26[25]$ & 27799 & 30303 & 297 & 399 & 102 \\
\hline & $n$-hexadecane & 2.33 & $29[25]$ & 27710 & 30303 & 297 & 391 & 94 \\
\hline
\end{tabular}

$1 \mathrm{cP}=10^{-3} \mathrm{~kg} /(\mathrm{m} \mathrm{s})=10^{-3} \mathrm{~Pa} \mathrm{~s}$.

${ }^{2}$ The error of the method: $\pm 10 \mathrm{~K}$.

Fig. 3. Absorption, fluorescence and $T^{*}(\tilde{v})$ spectra of erythrosin B in water, as well as the corresponding $F(\tilde{v})$ function. 

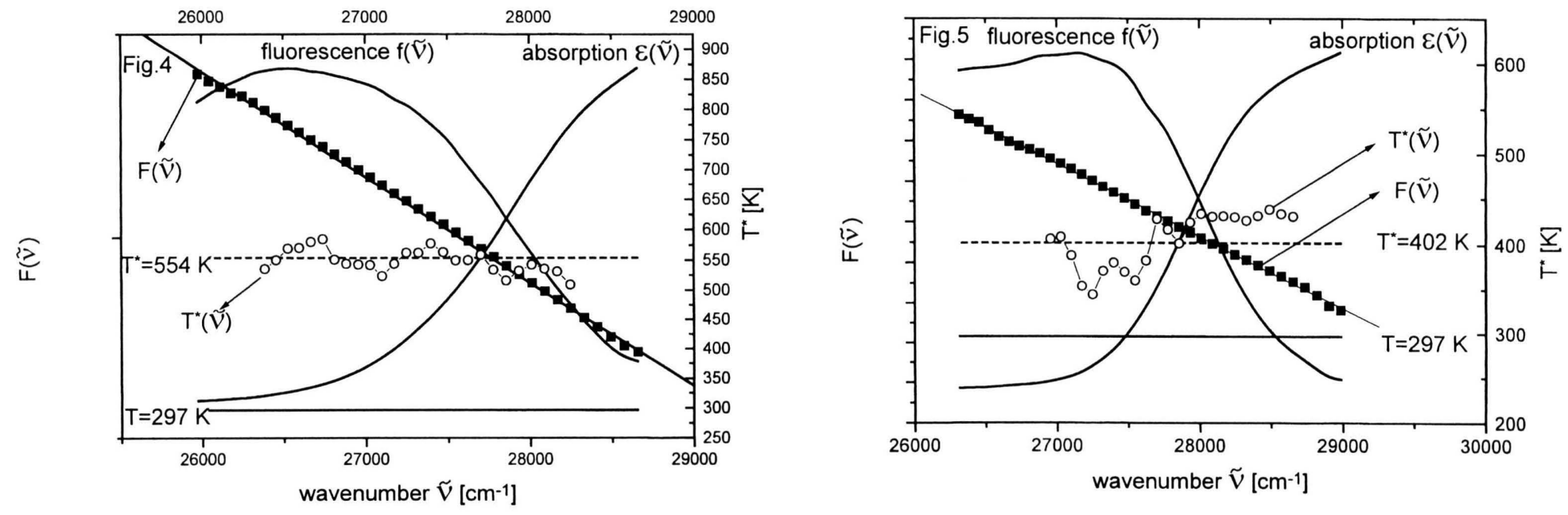

Figs. 4. and 5. Absorption, fluorescence and $T^{*}(\tilde{v})$ spectra of 2a and 3a in $n$-hexane, respectively, as well as the corresponding $F(\tilde{v})$ function.
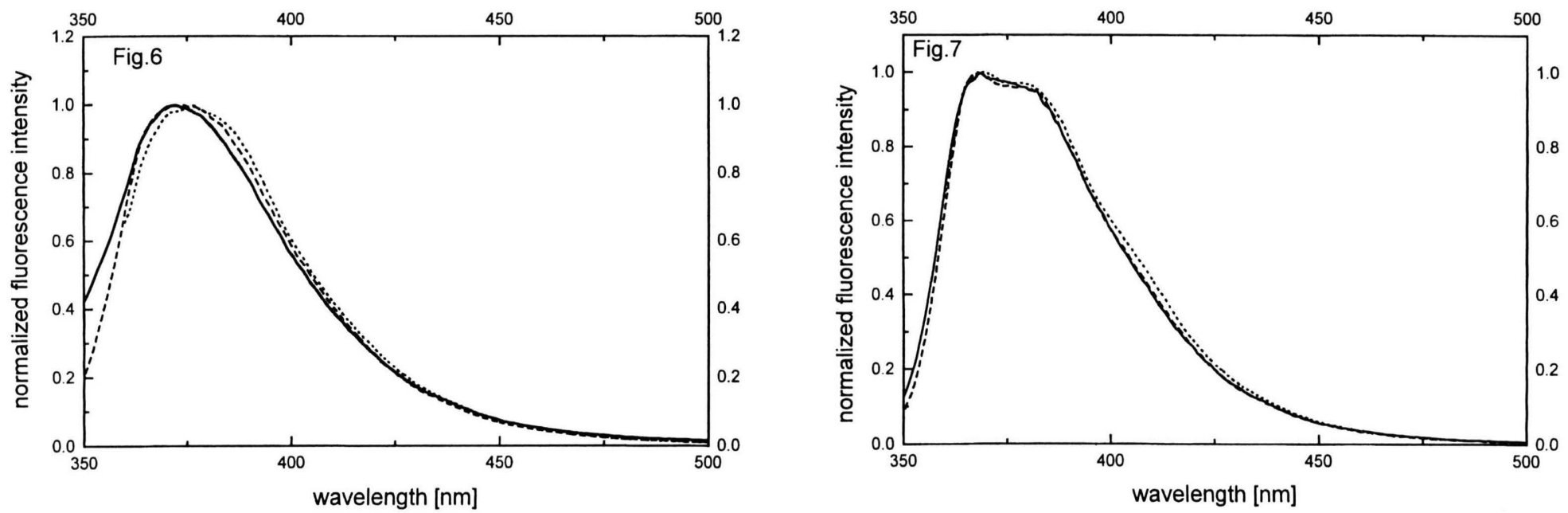

Figs. 6. and 7. Flourescence spectra of 2a and 3a in $n$-hexane, respectively, for different excitation wavelengths: $\lambda_{\text {exc }}=310 \mathrm{~nm}$ (solid line), $\lambda_{\text {exc }}=330 \mathrm{~nm}$ (dashed line) and $\lambda_{\text {exc }}=350 \mathrm{~nm}$ (dots). 


\section{Experimental}

4-dimethylamino- $\omega$-diphenylphosphinyl-trans-styrene (2a) and 4-dimethylamino- $\omega$-methylsulphonyltrans-styrene (3a) of spectroscopic grade were synthesized and kindly gifted by Dr. Dieter Gloyna (Humboldt Universität zu Berlin).

The dyes: rhodamine $B$, rhodamine $S$, rhodamine $6 \mathrm{G}$, Na-fluorescein, and erythrosin B analytically pure were additionally purified by multiple crystallization. All alkane solvents were spectroscopically pure. Water was doubly distilled. The absorption spectra were recorded on a fully computerized Zeiss model M-40 spectrophotometer and fully corrected fluorescence spectra were measured using a spectrofluorimeter designed and built in our laboratory [21].

\section{Results and Discussion}

We selected seven luminescent compounds, four of them with long lifetimes (several nonoseconds) and three with short lifetimes ranging (from 7 ps to 77 ps). Figs. 1 and 2 present the absorption and fluorescence spectra as well as spectral temperature $T^{*}(\tilde{v})$ and $F(\tilde{v})$ courses for rhodamine $\mathrm{B}$ and rhodamine $6 \mathrm{G}$, respectively, in water (a trace amount of $0.1 \mathrm{~N}$ solution of $\mathrm{HCl}$ was added to ensure the presence of a single ionic form). Once can observe a linear dependence of $F(\tilde{v})$ over the overlap region between the absorption and fluorescence band, which is consistent with (1). However, in the bands overlap a strong fluctuation of $T^{*}(\tilde{v})$ around the horizontal line corresponding to the temperature of the experiment. Strong departures of this function from linearity appear only on the edges of the absorption and fluorescence bands (for example for rhodamine $B$ above $\tilde{v}=18400 \mathrm{~cm}^{-1}$ (fluorescence band) and below $\tilde{v}=17100 \mathrm{~cm}^{-1}$ (absorption band). A similar behaviour is found for rhodamine $\mathrm{S}$ and Na-fluorescein in water. Temperatures $T^{*}$ determined based on (2) are listed in Table 1. It can be seen that the differences $\Delta T=T^{*}-T$ are within the limits of experimental error $\pm 10 \mathrm{~K}$ (resulting from the uncertain-

[1] E. H. Kennard, Phys. Rev. 11, 29 (1918).

[2] E. H. Kennard, Phys. Rev. 28, 672 (1926).

[3] B. J. Stepanov, DAN SSSR 112, 839 (1957).

[4] B. J. Stepanov, IAN SSSR 22, 1034 (1958); 22, 1367 (1958).

[5] M. N. Alentsev, Opt. Spektrosk. 4, 690 (1958).

[6] A. Schmillen and R. Legler, Vol. 3, Luminescence of Organic Substances, in Landoldt-Börnstein, Numerical Data and Functional Relationships in Science and Technology, ty of the slope of $F(\tilde{v}))$. Our results are in a full agreement with the earlier ones obtained for nonpolar and polar luminescent molecules in nonpolar and polar solvents $[15-17,22]$. It has been found therein that the polarity of both the luminescent and solvent molecules does not exhibit a significant dependence on the local temperature.

A completely different behaviour, compared to the mentioned dyes, was found in this work for other luminescent compounds, the lifetimes of which are very short. Figures 3-5 show the results of investigations on erythrosin $\mathrm{B}$ in water and on $2 \mathrm{a}$ and $3 \mathrm{a}$ in $n$-hexane, respectively. The linear dependence of $F(\tilde{v})$ according to (2) is fulfilled over the absorption and fluorescence overlap range, however the temperatures $T^{*}$ determined from the slope of $F(\tilde{v})$ exceed distinctly the ambient temperature $T$ (cp. Table 1). In Figs. 3-5 the function $T^{*}(\tilde{v})$ is plotted only over such a range of absorption and fluorescence where no significant experimental error is made.

Data obtained for these short lived solutes suggest that higher values of $T^{*}$ are caused by the non-establishment of statistical equilibrium before the emission act over the vibrational levels of excited molecules. In this case part of the excess of excitation energy is not dispersed throughout the solvent during the short lifetime of the solute. This is the reason of the high local temperature $T^{*}$ which holds also during the emission process. In view of the very short lifetimes of the molecules studied ( 7 ps-77 ps) and the lack of thermal equilibrium with the surrounding medium it should be expected that the intensity distribution in the emission band as well as its spectral location will not be independent of the excitation light frequency. Figures 6 and 7 show that indeed a slight dependence of the fluorescence band on the excitation light frequency is observed.

\section{Acknowledgement}

We would like to thank the DAAD for a gift of MCP photomultiplier. This work has been supported by the grants BW 5200-5-0004-9. We would like to thank Professor Wiesław Wiczk for using his absorption and fluorescence equipment to test some of our results.

New Series, Editor K.-H. Hellwege, Springer Verlag, Berlin 1967 (here a review of earlier literature).

[7] B. S. Neporent, N. G. Bakshiev, and J. T. Mazurenko, in "Elementary Photoprocesses in Molecules" (in Russian), Izd. Nauka, Moskva 1966, p. 80.

[8] J. Hevesi and L. Kozma, Acta Phys. Chem. Szeged 8, 103 (1962).

[9] I. Ketskemety, L. Kozma, and J. Hevesi Acta Phys. Chem. Szeged 12, 83 (1966). 
[10] L. Kozma, L. Szalay, and J. Hevesi, Acta Phys. Chem. Szeged 10, 68 (1964).

[11] J. Hevesi and L. Kozma, and L. Szalay, Acta Phys. Polon. 29, 57 (1966).

[12] W. P. Klotshkov and S. M. Korotkov, Opt. Spektrosk. 22, 345 (1967).

[13] N. G. Bakshiev, I. W. Piterskaya, W. I. Studentov, and A. W. Altayskaya, Opt. Spektrosk. 27, 349 (1969).

[14] N. G. Bakshiev, I. W. Piterskaya, and A. W. Altayskaya, Opt. Spektrosk. 28, 897 (1970)

[15] A. Kawski and J. Kukielski, Z. Naturforsch. 25a, 653 (1970).

[16] A. Kawski, B. Bartoszewicz, and J. Kukielski, Acta Phys. Polon. A38, 127 (1970).

[17] A. Kawski and J. Kukielski, J. Luminescence 4, 155 (1971).
[18] A. Kawski, Z. Naturforsch. 39a, 509 (1984).

[19] D. A. Sawicki and R. S. Knox, Phys. Rev. A 54, 4837 (1996).

[20] R. S. Knox, Acta Phys. Polon. A 95, 85 (1999).

[21] A. Kawski, G. Piszczek, B. Kukliński, and T. Nowosielski, Z. Naturforsch. 49a, 824 (1994).

[22] A. Kawski and J. Kukielski, Z. Naturforsch. 25a, 1380 (1970).

[23] T. Lopez Arbeloa, M. J. Tapia Estevez, F. Lopez Arbeloa, I. Urretxa Aquirresacona, and I. Lopez Arbeloa, J. Luminescence 48\&49, 400 (1991).

[24] A. Matczuk, P. Bojarski, I. Gryczynski, J. Kusba, L. Kulak, and C. Bojarski, J. Photochem. Photobiol. A 90, 91 (1995).

[25] A. Kawski, I. Gryczynksi, K. Nowaczyk, P. Bojarski, and J. Lichacz, Z. Naturforsch. 46a, 1043 (1991). 